\title{
Study on the Growth Mechanism of Intermetallic Compounds in Solid Interface of Aluminum / Steel
}

\author{
Hengbo Ma ${ }^{1, a}$, Ranfeng Qiü,b ,Jiuyong Liu ${ }^{3, \mathrm{c}}$ and Yangyang Zhao,d \\ ${ }^{1}$ School of Materials Science and Engineering, Henan University of Science and Technology, \\ Luoyang, 471003, China \\ ${ }^{2}$ Henan Key Laboratory of Advanced Non-ferrous Metals, Henan University of Science and \\ Technology, Luoyang 471003, China \\ ayimin@mail.haust.edu.cn, bxdqrf@yahoo.com.cn, chyhx66@163.com, dzhangxin_sdha@163.com
}

Keywords: explosive welding, aluminum / steel ,the intermetallic compound, thickness.

Abstract. In this experiment, the intermetallic compound of pure aluminum / Q235 low carbon steel was studied by explosive welding. The thickness of IMC in aluminum / steel interface under different heat treatment temperature and heat treatment time was studied. It is found that the thickness of the intermetallic compound layer is different under different holding time, the longer the heating time is, the larger the thickness is. This is because in the constant temperature conditions, although the diffusion coefficient remains unchanged, but with the heating time, atomic diffusion is sufficient to make the intermetallic compound to grow and thicker.

\section{Introduction}

Recently, the frequent occurrence of haze phenomenon has seriously affected the daily lives of residents, the study shows that reducing the weight of the car itself can effectively solve the problem. The data show that the car's own weight for each $10 \%$ lower, fuel consumption will be reduced by $6 \%$ to $10 \%$, so the exhaust emissions will be a corresponding reduction of $4 \%{ }^{[1]}$. The main means of vehicle weight reduction is to replace the steel plate in the body into a lighter aluminum alloy and other materials, aluminum and steel physical and chemical properties of the comparison that: the welding between the two is poor, but the two The connection between them is essential. Therefore, for the aluminum alloy and steel is not easy to weld this problem ${ }^{[2-4]}$, domestic and foreign researchers on the aluminum / steel dissimilar materials between the connection has been extensively studied. While the study of the intermetallic compound layer in the solid state steel / solid aluminum state is still rarely reported.

\section{Experimental Procedure}

In this experiment, Q235 low carbon steel plate and pure aluminum plate were used as the welding base metal, among which pure aluminum was used as the cladding plate and Q235 steel as the substrate. The thickness of pure aluminum plate is $15 \mathrm{~mm}$, and the thickness of steel plate is $20 \mathrm{~mm}$ and $40 \mathrm{~mm}$ respectively.The cleaning steps of the pre-weld sample were as follows:

(1) the use of rasp to remove pure aluminum and low carbon steel Q235 board of the flash, and the use of wire brushing the surface of the base material to remove the surface of the oxide film, corrosion and other impurities.

(2) Then use alcohol or acetone to scrub the surface of the base oil, scrub clean with a hair dryer.

The joints of Q235 low carbon steel and pure aluminum heterogeneous materials were studied by using the explosive welding method as shown in the figure.1. The process parameters used in the explosion welding are as follows: parallel installation method, low-speed rock ammonium nitrate explosives, the basis of the ratio were 2.67 and 1.33 . One of the aluminum plate for the web, steel for the substrate. 


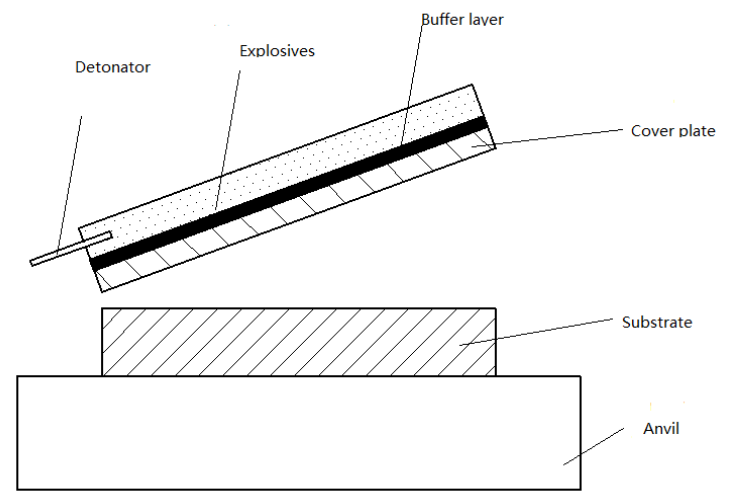

Figure.1 Schematic diagram of explosion welding

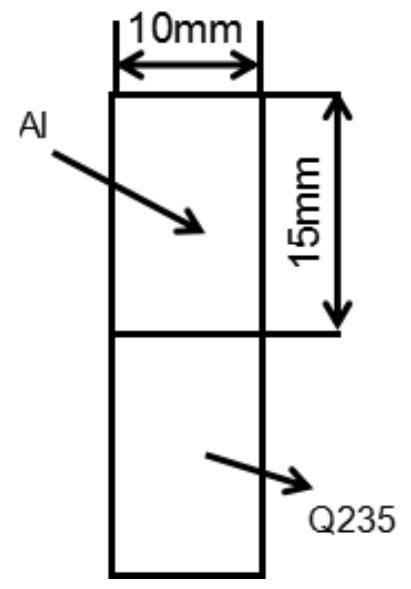

Fig.2 The schematic diagram

The sawing and thread cutting treatments yielded a schematic diagram for tissue observation as shown in Fig.2.Then a set of samples after thread cutting was placed in a thermostatic chamber for heat treatment. According to the melting point of aluminum, in the case of ensuring that the interface is solid, the incubator temperature $\mathrm{T}$ used in this study is $600{ }^{\circ} \mathrm{C}, 500{ }^{\circ} \mathrm{C}, 400{ }^{\circ} \mathrm{C}, 300{ }^{\circ} \mathrm{C}$. When the temperature remains the same, the heating time is $1,2,4,9$, and $16 \mathrm{~h}$, respectively. In this study, each test sample was 5 , of which 2 were used for tissue observation and 3 for tensile tests. The specific heat treatment process is that the thermostat instrument open and set the appropriate parameters of heat treatment, the temperature in the oven temperature reaches the set temperature experiment; in the sample and record the time; when the holding time reaches the required test, with pliers will be placed in the outside, the asbestos tile in natural cooling room temperature.

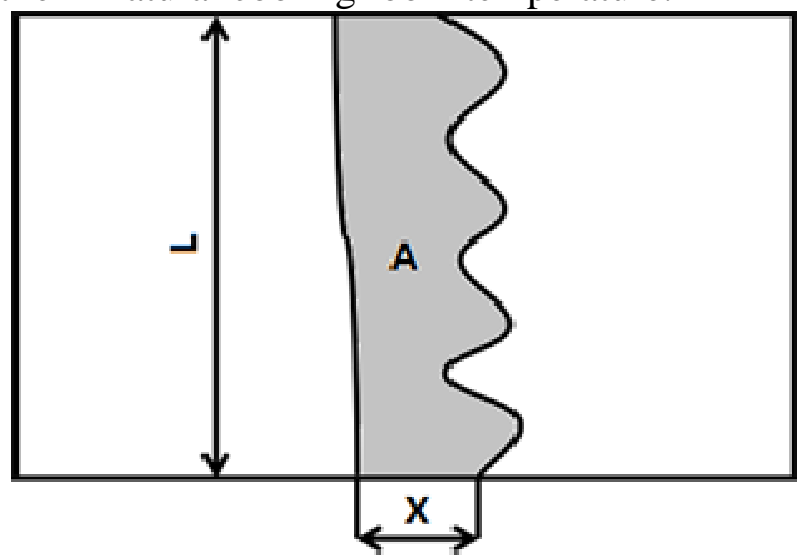

Fig.3 The measuring schematic diagram of IMC thickness

The change in the thickness of the intermetallic compound layer at the interface of the aluminum / steel joint is studied, so the measurement of the thickness of the intermetallic compound is essential. In order to accurately determine the change in the thickness of the intermetallic compound layer, the average thickness of the intermetallic compound is measured, and the measurement method used is shown in Fig. 3. Where $\mathrm{A}$ is the area of the gray area and $\mathrm{L}$ is the length of the gray, so that the average thickness $\mathrm{X}$ of the intermetallic compound is the ratio of A to L.

\section{Experimental results and discussion}

Figure 4 (a), (b), and (c) show the scanning electron microscopy of the interface of the aluminum / steel joint specimen at a heating time of $1 \mathrm{~h}, 2 \mathrm{~h}$, and $16 \mathrm{~h}$ at $600^{\circ} \mathrm{C}$. As shown in the figure, the thickness of the intermetallic compound layer is different under different holding conditions, and the longer the heating time is, the larger the thickness is. This is because, under the condition of constant temperature, although the diffusion coefficient remains unchanged, the diffusion of atoms increases sufficiently with the heating time, and the intermetallic compound is sufficiently grown to grow thicker. 


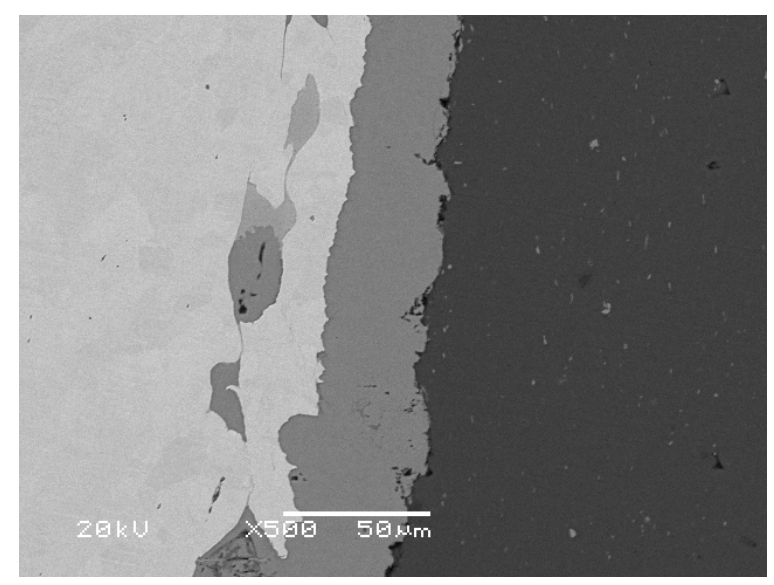

(a) Heating time $1 \mathrm{~h}$

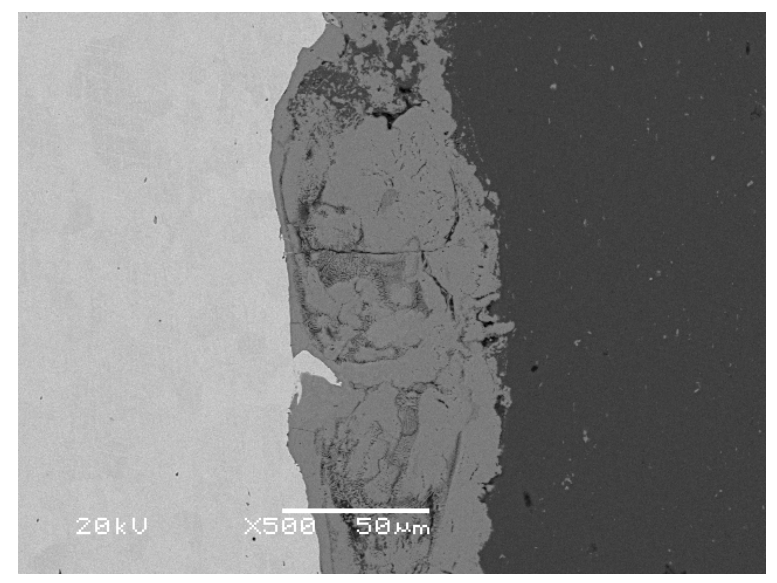

(b) Heating time $2 \mathrm{~h}$

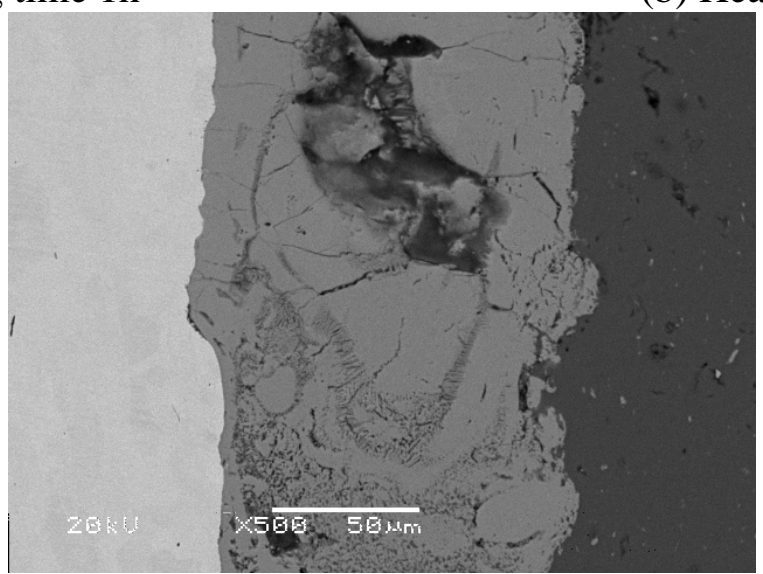

(c) Heating time 16h

Fig. 4 The scan electron picture under the condition of different heat treat time

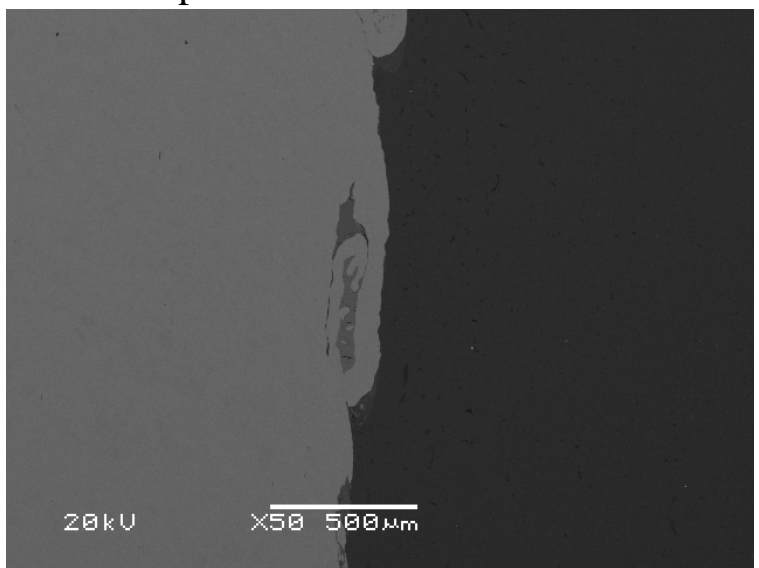

Fig. 5 The low power scan electron picture of aluminum and steel interface

The micro-zigzag morphology of the intermetallic compound was not observed on the steel side as compared to the aluminum / steel explosive welded joint interface without heat treatment shown in Figure 5. The intermetallic compound morphology is also different from other welding methods of the interface interface "tongue" intermetallic compounds ${ }^{[5]}$, both sides of the shape is more straight. This is because when the holding time is long enough, the interdiffusion between the aluminum and iron atoms will be more fully, as described later, the intermetallic compound grows homogenized.

In addition, the presence of defects such as cracks and pits was observed in the intermetallic compounds shown in Figs. 4 (b) and (c). The reason for this is that the linear expansion coefficient of aluminum is $23.6 \times 10-6 \cdot \mathrm{K}^{-1}$ and the thermal expansion coefficient of steel is $11.76 \times 10-6 \cdot \mathrm{K}^{-1}$. During the cooling process, the two kinds of base materials are not synchronized, resulting in the intermediate intermetallic compound layer being subjected to tensile stress on the steel side, resulting 
in the generation of cracks. The pits are produced because the intermetallic compounds are relatively brittle, there are cracks inside, in the sample grinding process of local loss.

\section{Conclusions}

The thickness of interfacial intermetallic compound layer is different under different holding time, the longer the heating time is, the larger the thickness is. This is because, under the condition of constant temperature, although the diffusion coefficient remains unchanged, the diffusion of atoms increases sufficiently with the heating time, and the intermetallic compound is sufficiently grown to grow thicker.

\section{References}

[1] Industrial Development Research Center of the State Council Development Research Center: 2010 China Automotive Industry Development Report, 2010.

[2] Elrefaey Ahmed, Takahashi Makoto, Ikeuchi Kenji. Cushing Society Proceedings, 2005,23 (2): 186-193.

[3] Fu Li, Mao Xinfu, Shi Xuefang. 2003 (24): 9-13.

[4] Zhang Changqing, Wang Xijing, Li Boqiang, et al . Journal of Lanzhou University of Technology, 2010,36 (6): 25-28.

[5] Qiu Ranfeng, Iwamoto Chihiro, Satonaka Shinobu. Materials Characterization, 2009,60(2):156-159. 